\title{
Polarimetric properties of aerosol particles
}

\author{
Itaru Sano and Sonoyo Mukai \\ Faculty of Science and Technology, Kinki University, 3-4-1 Kowakae, Higashi-Osaka 577-8502, Japan
}

(Received October 7, 1997; Revised December 17, 1997; Accepted January 20, 1998)

\begin{abstract}
Retrieval algorithms for scattering particles are shown based on photopolarimeric measurements of sky light over the ocean and multiple scattering simulations of the polarization field. Polarized components of the atmospheric constituents have been measured by a photopolarimeter (named PSR1000) with spectral bands set up to correspond to the ADEOS/POLDER. The POLDER is the first sensor on board the satellite to be designed to observe polarization.

It is shown that heterogeneous grains are better than homogeneous models to explain polarimetric properties of atmospheric aerosols, and a Maxwell-Garnett mixing rule for small water-soluble (WS) inclusions in an oceanic (OC) matrix is available to interpret the polarization measurements of atmospheric aerosols over the Seto Inland Sea. We also found during our observations that the value of refractive index of the aerosol, i.e., its chemical composition, varies with time and place rather than particle size.
\end{abstract}

\section{Introduction}

It is well-known atmospheric aerosols play an important role in the earth's environment. Unfortunately, aerosol characteristics may change greatly in temporal and regional scales as shown by Liu et al. (1996), Dalu et al. (1995), and Pilinis et al. (1995). Further as for the remote sensing of surface and ocean, the aerosol characteristics in addition to the optical thickness are required for atmospheric correction (Mukai et al., 1992). At present, there are several aerosol radiative models, but these are rather based on measurements over limited areas. Thus it is our purpose to continue our aerosol measurements and simultaneously develop efficient algorithms to retrieve aerosol characteristics (Porter and Clarke, 1997; Tanré et al., 1996).

It has been shown in our previous work (Mukai et al., 1996) that polarization features strongly depend on the aerosol model, and that aerosol retrieval can be accurately pursued by combining polarization with radiance. Therefore shipboard measurements of sky light with a portable photopolarimeter are used for aerosol retrieval based on multiple scattering simulations of the polarization field.

\section{Polarimetric Properties of Heterogeneous Grains}

The polarimetric observations were undertaken with a portable photopolarimeter (PSR1000, Opto Research Co.) during a research cruise with Fukaemaru of the Maritime University of Kobe around Shikoku and Kyushu islands of Japan from July 23 to 30 in 1996, and in the Seto Inland Sea on March 18 in 1997 in clear atmospheric conditions. PSR1000 has the observing bands at wavelengths of $0.443,0.490$, $0.565,0.670,0.765$, and $0.865 \mu \mathrm{m}$ and a Glan-Thompson prism is attached in front of the radiometer of PSR1000 as polarizer, rotated manually to $90^{\circ}$ away from the solar direc-

Copy right (C) The Society of Geomagnetism and Earth, Planetary and Space Sciences (SGEPSS); The Seismological Society of Japan; The Volcanological Society of Japan; The Geodetic Society of Japan; The Japanese Society for Planetary Sciences. tion in the principal plane determined by the sun, zenith and observation directions. Figures 1 and 2 show the trajectory of measurements and observed polarization degree.

Roughly speaking, the degree of polarization decreases with wavelength in almost all cases in the near infrared region, but in the Inland Sea it shows a steeper decrease than in the Pacific Ocean. In other words, the observed results are divided into two groups with respect to wavelength dependency. That is, the difference of trend represents the difference in aerosol characteristics.

A procedure for aerosol retrieval by using photopolarimetric measurements has been shown by Mukai et al. (1996). We obtained the following conclusions based on light scattering calculations in respect of aerosol models;

(1) the typical oceanic type aerosol is one candidate for an aerosol model to investigate the photopolarimetry over the sea,

(2) it is more natural to consider that the continental type aerosol exists together with the oceanic type.

In those simulations the aerosols are assumed to be homogeneous, but it is natural to consider that several kinds of aerosol components could exist together in general.

In this section, we take into account heterogeneous particles for maritime aerosols. The World Meteorological Organization (WCP-112, 1986), provides a standard maritime aerosol model, which is mainly composed of oceanic (OC) and water-soluble (WS) components. "Oceanic" denotes sea-salt solution in water, and "water-soluble" includes water-soluble substances consisting of ammonium sulfate, calcium sulfate and organic compounds. We assume now that the aerosols are composed of an oceanic component and a water-soluble component.

The mixing rules of several components into spherical shaped heterogeneous particles have been presented by several authors (Bohren and Wickramasinghe, 1977; Chýlek and 


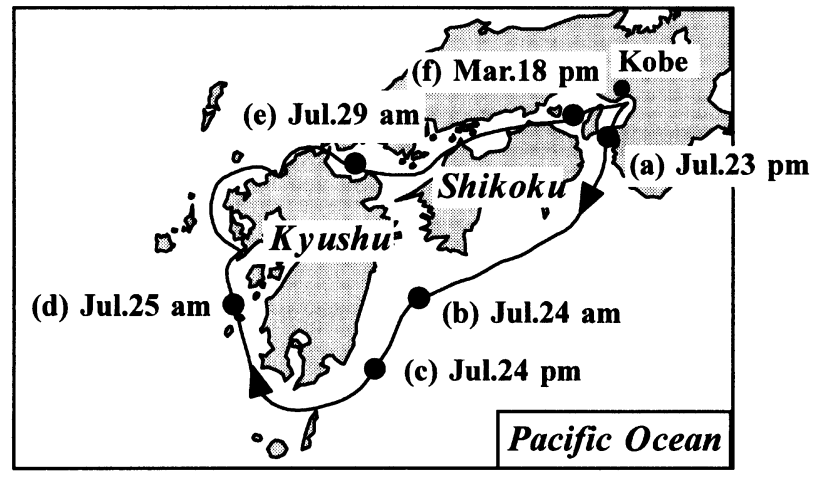

Fig. 1. The geographical positions of the observation sites, which are denoted by symbols on the map of Japan.

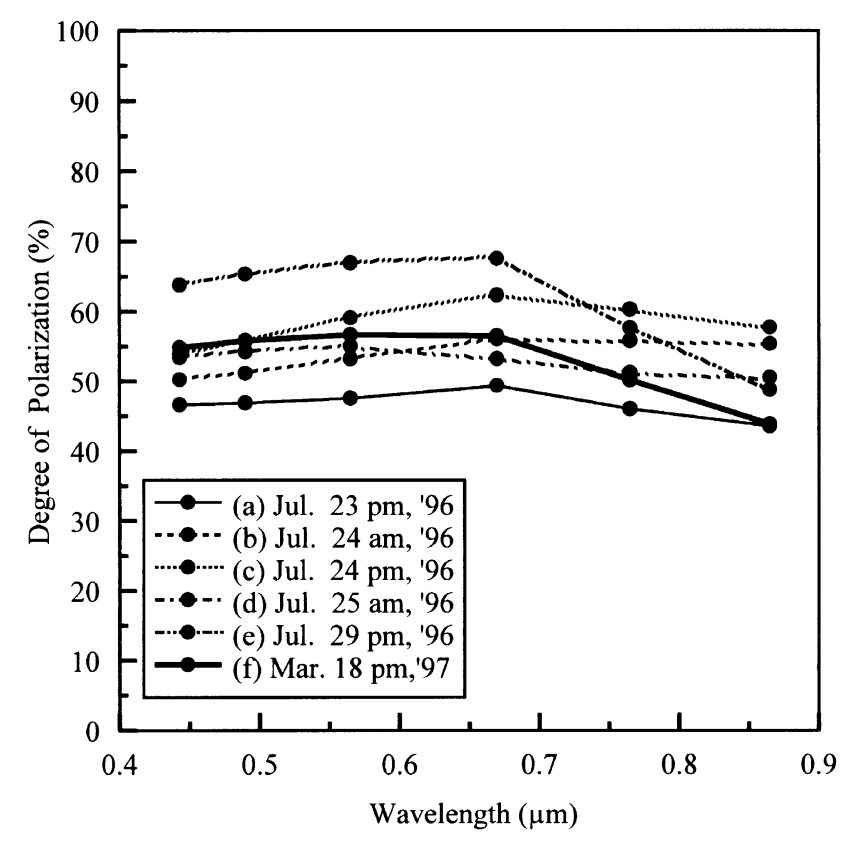

Fig. 2. Observed degree of polarization versus wavelength, where the dots represent observed data at the position of (a)-(f) in Fig. 1.

Srivastava, 1983; Burkhard, 1984). Our present simulations employ the Maxwell-Garnett (MG) mixing rule. So brief outline of MG theory is introduced here. Consider the averaged dielectric function $\epsilon_{\mathrm{av}}$ of a heterogeneous particle consisting of small spherical particles (called inclusions) suspended in a homogeneous medium (called matrix) is expressed as;

$$
\epsilon_{\mathrm{av}}=\epsilon_{\mathrm{m}} \frac{\left(\epsilon_{\mathrm{i}}+\epsilon_{\mathrm{m}}\right)+2 f_{\mathrm{i}}\left(\epsilon_{\mathrm{i}}-\epsilon_{\mathrm{m}}\right)}{\left(\epsilon_{\mathrm{i}}-\epsilon_{\mathrm{m}}\right)-f_{\mathrm{i}}\left(\epsilon_{\mathrm{i}}+\epsilon_{\mathrm{m}}\right)},
$$

where $\epsilon_{\mathrm{m}}$ and $\epsilon_{\mathrm{i}}$ are dielectric functions of the matrix and inclusions. A variable $f$ is the volume fraction of inclusions in the matrix. The relation between the dielectric function $\epsilon$ and the complex refractive index $m=n-i k$ is:

$$
\begin{aligned}
& \operatorname{Re}(\epsilon)=n^{2} k^{2}, \\
& \operatorname{Im}(\epsilon)=2 n k .
\end{aligned}
$$

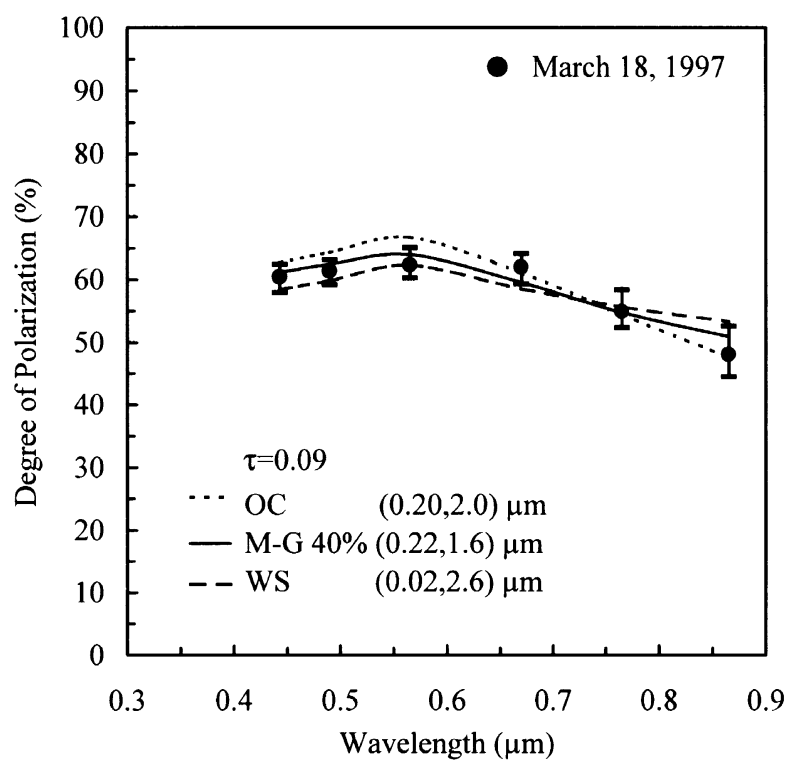

Fig. 3. The degree of polarization versus wavelength, where the dots represent observed data on March 18 in 1997 at the position of (f) in Fig. 1, and the solid, dotted and dashed curves denote simulated results by using heterogeneous aerosol model and OC- and WS-homogeneous ones, respectively.

Table 1 presents the complex refractive indices of heterogeneous particles, where the components of matrix and inclusions are OC and WS respectively. It is found from the table that the refractive index for a heterogeneous particle shows a proportional allotment to the value $f$.

Here a log-normal type is adopted for size distribution function for model aerosols. The function is expressed as follows;

$$
\frac{d N(r)}{d r}=\frac{N}{\sqrt{2 \pi} r \ln \sigma} \exp \left(-\frac{(\ln r-\ln \bar{r})^{2}}{2 \ln ^{2} \sigma}\right) .
$$

where $\bar{r}$ and $\sigma$ represent the most probable value of $\log r$ and the standard deviation of $\log r$. Thus it becomes our purpose to calculate the degree of polarization for the model aerosol with the value of refractive index given by above Eqs. (1)(3) and with the size distribution of the mode radius $(\bar{r} \mu \mathrm{m})$ and the width $(\sigma)$ of the log-normal curve based on multiple scattering calculations. A detailed description is shown in the next section.

A single scattering phase matrix $P$ is expressed by the Stokes parameters $(I, Q, U, V)$ with the following form;

$$
P(\Theta)=a P_{\mathrm{a}}(\Theta)+(1-a) P_{\mathrm{g}}(\Theta),
$$

where $\Theta$ is the scattering angle and subscripts ' $a$ ' and ' $g$ ' denote the aerosols and molecular gases respectively. The functions $P_{\mathrm{a}}$ and $P_{\mathrm{g}}$, respectively, are given by the Mie scattering phase matrix if an aerosol model is selected and by the Rayleigh phase matrix. The value of $a$ represents the ratio of the aerosol opacity to the total opacity. Namely the value of $a$ is derived from aerosol optical thickness. Furthermore optical thickness of the atomosphere plays an important role in multiple scattering calculations. An example 
Table 1. The complex refractive indices of heterogeneous particles in according to Maxwell-Garnett mixing rule with volume fraction $f$.

\begin{tabular}{ccccccc}
\hline $\begin{array}{c}f \\
\text { Wavelength }(\mu \mathrm{m})\end{array}$ & $0.0(\mathrm{OC})$ & 0.1 & 0.2 & 0.3 & 0.4 & $1.0(\mathrm{WS})$ \\
\hline 0.443 & 1.387 & $1.401-0.0005 i$ & $1.415-0.0010 i$ & $1.429-0.0014 i$ & $1.443-0.0019 i$ & $1.530-0.0050 i$ \\
0.490 & 1.385 & $1.400-0.0005 i$ & $1.414-0.0010 i$ & $1.428-0.0014 i$ & $1.442-0.0019 i$ & $1.530-0.0050 i$ \\
0.565 & 1.383 & $1.397-0.0006 i$ & $1.412-0.0012 i$ & $1.426-0.0017 i$ & $1.441-0.0023 i$ & $1.530-0.0060 i$ \\
0.670 & 1.380 & $1.394-0.0006 i$ & $1.409-0.0013 i$ & $1.424-0.0019 i$ & $1.439-0.0026 i$ & $1.530-0.0066 i$ \\
0.765 & 1.378 & $1.392-0.0009 i$ & $1.407-0.0018 i$ & $1.421-0.0026 i$ & $1.436-0.0035 i$ & $1.526-0.0091 i$ \\
0.865 & 1.375 & $1.389-0.0012 i$ & $1.403-0.0023 i$ & $1.418-0.0035 i$ & $1.432-0.0047 i$ & $1.520-0.0121 i$ \\
\hline
\end{tabular}

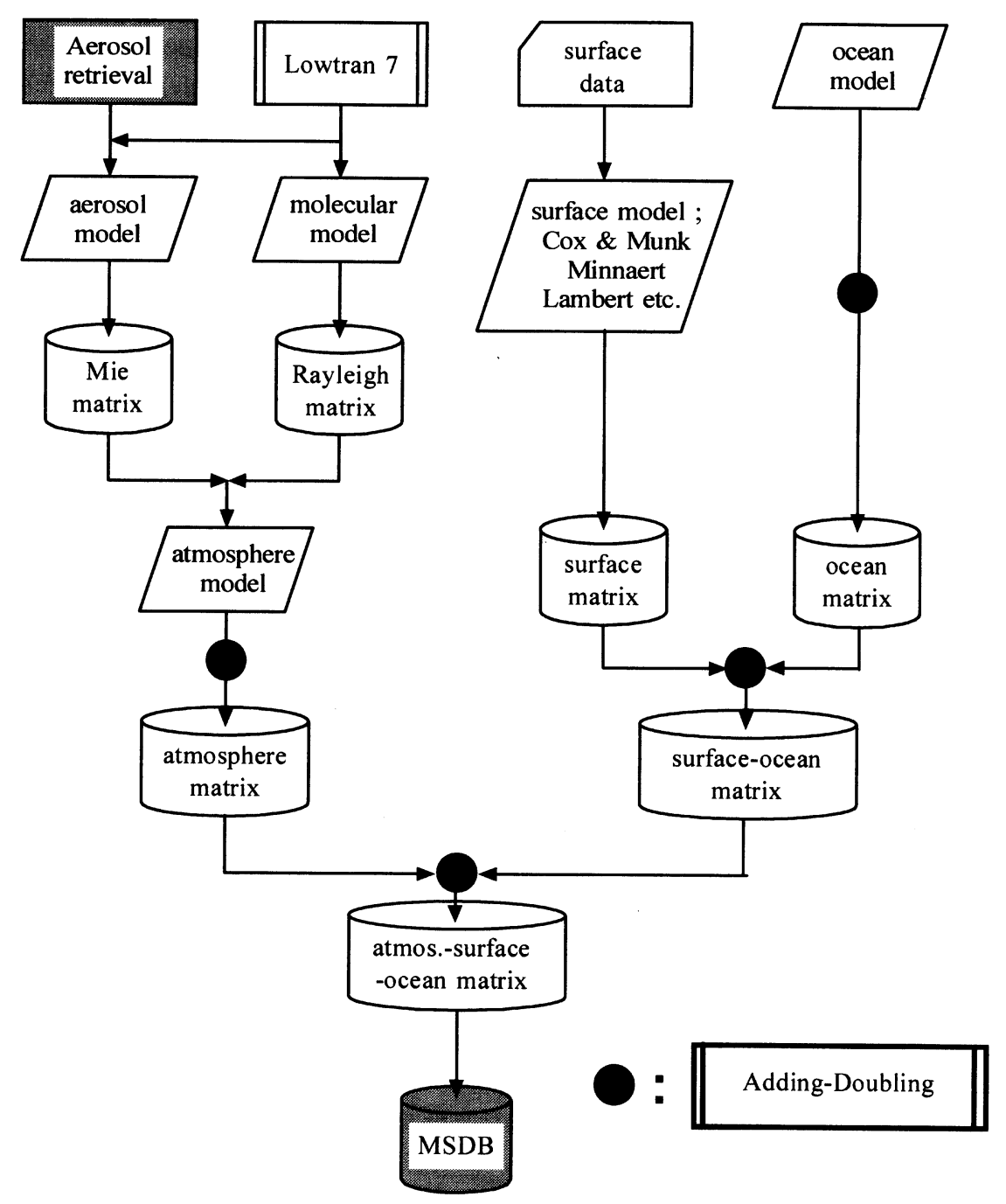

Fig. 4. A block flow chart of data and algorithms in our multiple scattering calculations in an atmosphere-sea surface-ocean model. Big filled circle represents adding-doubling procedure.

of aerosol retrieval using observed polarization degree, on March 181997 based on multiple scattering calculations, is presented in Fig. 3. The optical thickness and polarization of atmospheric constituents were simultaneously measured with PSR1000. We have found that the magnitude of polarization degree is reduced due to multiple scattered light, especially in the visible wavelengths (Sano et al., 1997). From this figure the following conclusions are drawn;

1) a water soluble type (WS) aerosol with $(r=0.1 \mu \mathrm{m}$, $\sigma=2.0 \mu \mathrm{m})$ and/or an oceanic type (OC) aerosol with $(r=0.24 \mu \mathrm{m}, \sigma=2.0 \mu \mathrm{m})$, which are denoted by the dotted and the dashed curves in Fig. 3, can explain the 
observed polarization degree,

2) a heterogeneous model with $\{f=0.4,(r=0.22 \mu \mathrm{m}$, $\sigma=1.6 \mu \mathrm{m})\}$ is a better candidate to explain the polarization data than homogeneous models.

\section{Multiple Scattering in an Atmosphere-Ocean Model}

Our algorithms for aerosol retrieval are based on multiple scattering calculations in an atmosphere-ocean model. A rough flow of our simulation procedure is shown in Fig. 4. An inhomogeneous plane-parallel atmosphere is simulated by four homogeneous sublayers $(0-5,5-13,13-25,25-100$ $\mathrm{km})$. The optical thickness of the Rayleigh scattering constituents and absorbent constituents such as ozone, water vapor and oxygen molecules are obtained by LOWTRAN 7 (Kneizys et al., 1988). Radiative transfer equation takes the following form

$$
\frac{\mu \partial I}{\partial \tau}=I(\tau, \Omega)-\int_{4 \pi} P\left(\Omega, \Omega^{\prime}\right) I\left(\tau, \Omega^{\prime}\right) d \Omega^{\prime} / 4 \pi,
$$

together with boundary conditions

$$
\begin{gathered}
I^{-}(0, \Omega)=\pi F_{0} \delta\left(\Omega-\Omega_{0}\right), \\
I^{+}\left(\tau_{1}+20, \Omega\right)=A / \pi \int_{2 \pi} I^{-}\left(\tau_{1}+10, \Omega^{\prime}\right) \mu^{\prime} d \Omega,
\end{gathered}
$$

where $\pi F_{0}$ represents incident solar net flux, and $I$ is the specific intensity expressed by the Stokes parameters $(I, Q, U$, $V$ ) at optical depth $\tau$ and in the direction of $\Omega$, which is represented by $\Omega=(\mu, \phi)$ and $d \Omega=d \mu d \phi$ by using cosine of polar angle $(\theta)$, i.e. $(\mu=\cos \theta)$, and azimuth angle $(\phi)$, and superscripts ' + ' and '- ' represent the upward and downward radiation field, respectively (see Fig. 5). The sea surface is simulated by multiple facets with slopes varying according to the isotropic Gaussian distribution with respect to wind speed (see Cox and Munk, 1954). Wind speed is assumed to be $5 \mathrm{~m} / \mathrm{sec}$ as a typical value for a clear day. Several ocean models (Hale and Querry, 1973; Morel, 1974; Tanaka and Nakajima, 1977; Masuda and Takashima, 1988) are available for our present calculations. The optical thickness of the ocean is fixed of 20 here. Multiple scattering for the atmosphere-ocean model is solved by the adding-doubling method.

We introduce the scattering and transmission matrices $S$ and $T$;

$$
\begin{aligned}
I^{+}(0, \Omega) & =\frac{1}{4 \pi \mu} S\left(\tau_{1}, \Omega, \Omega_{0}\right) \pi F_{0}, \\
I_{\mathrm{A}}^{-}\left(\tau_{1}, \Omega\right) & =\frac{1}{4 \pi \mu} T\left(\tau_{1}, \Omega, \Omega_{0}\right) \pi F_{0} .
\end{aligned}
$$

Matrices $S$ and $T$ are functions of optical thickness $\tau_{1}$ and angles $\Omega$ and $\Omega_{0}$. In terms of these matrices, we shall briefly describe the adding-doubling method (refer to Fig. 6). Assuming the matrices $S\left(x_{1}\right), T\left(x_{1}\right)$ and $S\left(x_{2}\right), T\left(x_{2}\right)$ for two sublayers of optical thickness $x_{1}$ and $x_{2}$, the scattering and transmission matrices for an adding layer of $\left(x_{1}+x_{2}\right)$ are given by

$$
S\left(x_{1}+x_{2}\right)=S\left(x_{2}\right)+\exp \left(-x_{2} / \mu\right) U+T\left(x_{2}\right) U
$$

$$
\begin{aligned}
T\left(x_{1}+x_{2}\right)= & \exp \left(-x_{1} / \mu\right) D \\
& +T\left(x_{1}\right) \exp \left(-x_{2} / \mu_{0}\right)+T\left(x_{1}\right) D
\end{aligned}
$$

$$
\begin{aligned}
Q_{1} & =S\left(x_{2}\right) S\left(x_{1}\right), \\
Q_{n} & =Q_{1} Q_{n-1}, \\
V & =\sum_{n=1}^{\infty} Q_{n}, \\
D & =T\left(x_{2}\right)+V \exp \left(-x_{2} / \mu_{0}\right)+V T\left(x_{2}\right), \\
U & =S\left(x_{1}\right) \exp \left(-x_{2} / \mu_{0}\right)+S\left(x_{1}\right) D .
\end{aligned}
$$

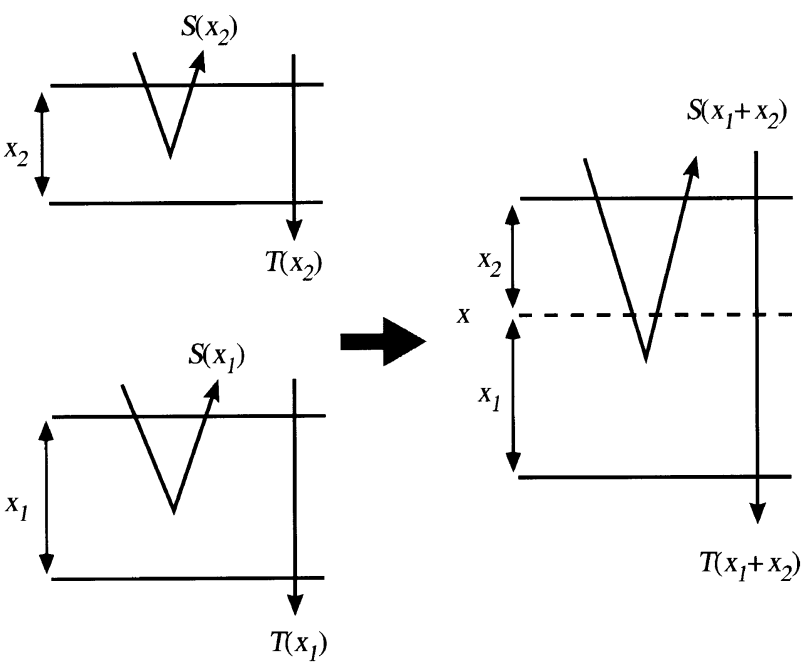

Fig. 5. An Illustration of radiative transfer model for atmosphere-sea surface-ocean system, where $I^{+}$and $I^{-}$represents the upward and downward specific intensity.

Fig. 6. Simple diagram of adding-doubling method, where $S$ and $T$ represent scattering and transmission matrices. 

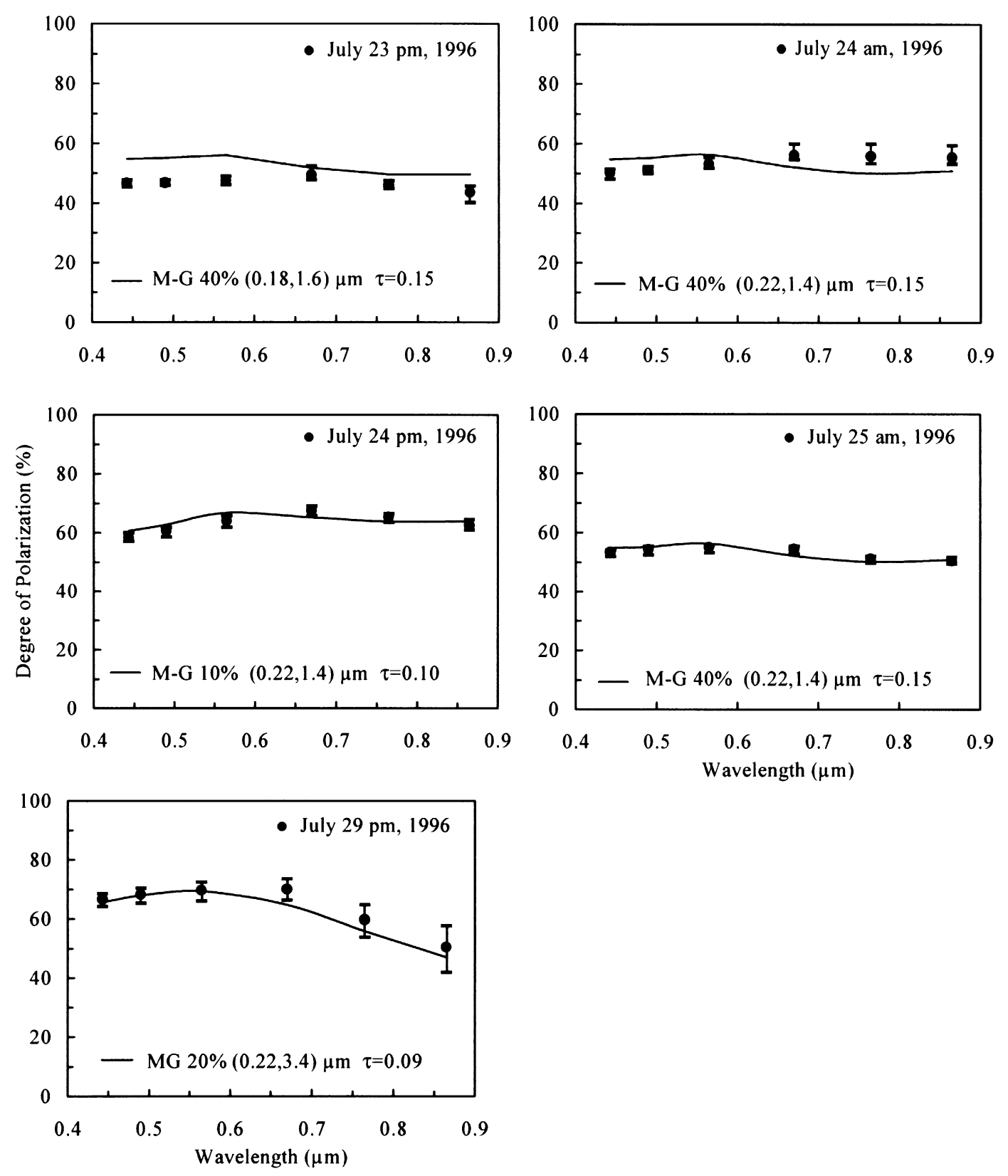

Fig. 7. The dots represent the observed polarization degree. The solid curves represent the numerical values for Maxwell-Garnett heterogeneous model based on multiple scattering calculations.

where the product of two matrices represents integration over the hemisphere as follows;

$$
A \cdot B=\frac{1}{4 \pi} \int_{0}^{2 \pi} \int_{0}^{1} A\left(\Omega, \Omega^{\prime}\right) B\left(\Omega^{\prime}, \Omega\right) \frac{d \mu^{\prime}}{\mu^{\prime}} d \phi^{\prime}
$$

In solving Eq. (6) based on the adding-doubling method, we should take into account the discontinuity of the radiation field at the sea surface level $\tau=\tau_{1}$. Once the scattering and transmission matrices have been obtained in the atmosphere and ocean alone, the matrices for the atmosphere-ocean system are derived from a superposition method (Mukai and
Ueno, 1978).

$$
\begin{aligned}
& U_{n}^{\mathrm{A}}=r_{\mathrm{A}} \cdot D_{n-1}^{\mathrm{A}}+t_{\mathrm{O}} \cdot U_{n-1}^{\mathrm{O}}, \\
& D_{n}^{\mathrm{O}}=t_{\mathrm{A}} \cdot D_{n-1}^{\mathrm{A}}+r_{\mathrm{O}} \cdot U_{n-1}^{\mathrm{O}}, \\
& D_{n}^{\mathrm{A}}=S_{\mathrm{O}} \cdot D_{n}^{\mathrm{O}}, \\
& U_{n}^{\mathrm{S}}=T_{\mathrm{A}} \cdot U_{n}^{\mathrm{A}}+\exp \left(-\tau_{1} / \mu\right) U_{n}^{\mathrm{A}} .
\end{aligned}
$$

where scripts $\mathrm{A}, \mathrm{O}$ and $\mathrm{S}$ denote atmosphere, ocean and atmosphere-ocean systems, respectively, $r$ and $t$ represent reflection and refraction matrices at the sea surface, $U$ and $D$ represent the upward and downward radiation fields, and $n$ is the number of interactions with the sea surface. Then 
the expected values in Fig. 5 are obtained as follows;

$$
\begin{aligned}
I^{+}(0, \Omega)= & \frac{1}{4 \mu} \sum_{n=0}^{\infty} U_{n}^{\mathrm{S}} \\
& +\pi F_{0} \exp \left\{-\tau_{1}\left(1 / \mu+1 / \mu_{0}\right)\right\} r_{\mathrm{A}}, \\
I_{\mathrm{A}}^{-}\left(\tau_{1}, \Omega\right)= & \frac{1}{4 \mu} \sum_{n=0}^{\infty} D_{n}^{\mathrm{A}} \\
& +\pi F_{0} \delta\left(\Omega, \Omega_{0}\right) \exp \left(-\tau_{1} / \mu\right), \\
I_{\mathrm{A}}^{+}\left(\tau_{1}, \Omega\right)= & \frac{1}{4 \mu} \sum_{n=0}^{\infty} U_{n}^{\mathrm{A}} \\
& +\pi F_{0} \delta\left(\Omega, \Omega_{0}\right) \exp \left(-\tau_{1} / \mu\right) r_{\mathrm{A}}, \\
I_{\mathrm{O}}^{-}\left(\tau_{1}, \Omega\right)= & \frac{1}{4 \mu} \sum_{n=0}^{\infty} D_{n}^{\mathrm{O}}+\pi F_{0} \exp \left(-\tau_{1} / \mu_{0}\right) t_{\mathrm{A}}, \\
I_{\mathrm{O}}^{+}\left(\tau_{1}, \Omega\right)= & \frac{1}{4 \mu} \sum_{n=0}^{\infty} U_{n}^{\mathrm{O}} .
\end{aligned}
$$

The measurements are compared with the desired value $I_{\mathrm{A}}^{-}\left(\tau_{1}, \Omega\right)$ for various aerosol models as mentioned in the previous section. Thus an optimized aerosol model is retrieved and the degrees of polarization are shown by the solid curves in Fig. 7. It is found from these figures that the volume fraction of inclusions to grain matrix varies with time and place, and the optical thickness of the atmosphere is also changing. We can say that the value of refractive index in the aerosol model, i.e., its chemical composition, rather than its size varies during our observations.

\section{Conclusions}

Photopolarimetric measurements are examined, based on multiple scattering calculations in the atmosphere-ocean system, and including heterogeneous aerosol models. This is a milestone for aerosol retrieval, because, until now, single scattering simulations using homogeneous grain model have been usually treated for interpretation of measurements of atmospheric aerosols.

In this work we obtain the following conclusions;

(1) heterogeneous grain models are better than homogeneous models to explain the polarimetric properties of atmospheric aerosols,

(2) A Maxwell-Garnett mixing rule as small water-soluble (WS) inclusions in an oceanic (OC) matrix is available to interpret the polarization measurements of atmospheric aerosols over the ocean near Japan,

2)-1 the volume fraction of inclusions to grain matrix varies with time and place,

2)-2 during our observations the chemical composition of the aerosol was more changeable than its size.

(3) The magnitude of polarization degree is reduced, due to multiple light scattering, especially at visible wavelengths.

(4) The optical thickness of the atmosphere is a key factor for interpretation of the photopolarimetry of sky light.

The observing wavelength of PSR1000 is designed to cor- respond to the ADEOS/POLDER. The POLDER (POLarization and Directionality of Earth Reflectance) is one of the sensors on board the satellite ADEOS and aims to collect globalscale visible and near-infrared observations of polarized and directional solar radiance reflected by the Earth-atmosphere system (Deschamps et al., 1994). Algorithms proposed in this work and measurements themselves are available to interpret the data given by ADEOS/POLDER.

Acknowledgments. The authors wish to express their thanks to Dr. R. Nakamura of Kobe University for his valuable comments on this study. The authors also wish to thank Prof. H. Ishida and cruise members on Fukaemaru of Maritime University of Kobe. This work partially supported by the Ministry of Education, Science and Culture of Japan $(09780485,09227223)$ and the NASDA (PSPC20170)

\section{References}

Bohren, C. F. and N. C. Wickramasinghe, On the computation of optical properties of heterogeneous grains, Astrophys. Space Sci., 50, 461-472, 1977.

Burkhard, D. G., Frequency dependent dielectric constant and conductivity for a medium containing impurities, Appl. Opt., 23, 2718-2727, 1984.

Chýlek, P. and V. Srivastava, Dielectric constant of a composite inhomogeneous medium, Phys. Rev. B, 27, 5098-5106, 1983.

Cox, C. and W. Munk, Measurements of the roughness of the sea surface from photographs of the sun's glitter, J. Opt. Soc. Amer., 44, 838-850, 1954.

Dalu, G., R. Rao, A. Pompei, P. Boi, G. Tonna, and B. Olivieri, Aerosol optical properties retrieved from solar aureole measurements over southern sardinia, J. Geophys. Res., 100, 26135-26140, 1995.

Deschamps, P. Y., F. M. Bréon, M. Leroy, A. Podaire, A. Bricaud, J. C. Buriez, and G. Sèze, The polder mission: Instrument characteristics and scientific objectives, IEEE Trans. Geosci. Remote Sensing, 32, 598-615, 1994.

Hale, G. M. and M. R. Querry, Optical constants of water in the 200-nm to 200- $\mu \mathrm{m}$ wavelength region, Appl. Opt., 12, 555-563, 1973.

Kneizys, F. X., E. P. Shettle, L. W. Abreu, J. H. Chetwynd, G. P. Anderson, W. O. Gallery, J. E. A. Selby, and S. A. Clough, Users guide to LOWTRAN 7, Tech. Rep. AFGL-TR-88-0177, Air Force Geophysics Laboratory, Hanscom AFB. MA, 1988.

Liu, P. S. K., W. R. Leaitch, C. M. Banic, S.-M. Li, D. Ngo, and W. J. Megaw, Aerosol observations at chebogue point during the 1993 north atlantic regional experiment: Relationships among cloud condensation nuclei, size distribution, and chemistry, J. Geophys. Res., 101, 2897128990, 1996.

Masuda, K. and T. Takashima, Dependence of the radiation just above and below the ocean surface on atmospheric and oceanic parameters, Appl. Opt., 27, 4891-4898, 1988.

Morel, A., Optical properties of pure water and pure sea water, in Optical Aspects of Oceanography, edited by N. G. Jerlov and E. S. Nielsen, 1 pp., Academic Press, London, 1974.

Mukai, S. and S. Ueno, Apparent contrast of an atmosphere-ocean system with an oil polluted sea surface, Appl. Math. Modelling, 2, 254-260, 1978 .

Mukai, S., I. Sano, K. Masuda, and T. Takashima, Atmospheric correction for ocean color remote sensing: Optical properties of aerosols derived from czcs imagery, IEEE Trans. Geosci. Remote Sensing, 30, 818-824, 1992.

Mukai, S., I. Sano, and T. Takashima, Investigation of atmospheric aerosols based on polarization measurements and scattering simulations, Opt. Rev., 3, 487-491, 1996

Pilinis, C., S. Pandis, and J. Seinfeld, Sensitivity of direct climate forcing by atmospheric aerosols to aerosol size and composition, J. Geophys. Res., 100, 18739-18754, 1995.

Porter, J. N. and A. D. Clarke, Aerosol size distribution models based on in situ measurements, J. Geophys. Res., 102, 6035-6045, 1997.

Sano, I., S. Mukai, M. Yasumoto, K. Masuda, M. Sasaki, and H. Ishida, Interpretation of ground- and space-based measurements of atmospheric aerosols, in Proc. IEEE IGARSS'97, pp. 9-11, 1997.

Tanaka, M. and T. Nakajima, Effects of oceanic turbidity and index of refraction of hydrosols on the flux of solar radiation in the atmosphere- 
ocean system, J. Quant. Spectrosc. Radiat. Transfer, 18, 93-111, 1977. Tanré, D., M. Herman, and Y. J. Kaufman, Information on aerosol size distribution contained in solar reflected spectral radiances, J. Geophys. Res., 101, 19043-19060, 1996.

World Climate Programme, WCP-112, A preliminary cloudless standard atmosphere for radiation computation, WMO/TD-No. 24, World Meteorological Organization, Geneva, 1986.

I. Sano (e-mail: sano@im.kindai.ac.jp) and S. Mukai 